\title{
Validation of the 4B5 rabbit monoclonal antibody in determining Her2/neu status in breast cancer
}

\author{
Bert van der Vegt ${ }^{1}$, Geertruida H de Bock ${ }^{2}$, Joost Bart ${ }^{1}$, Nick G Zwartjes ${ }^{1}$
} and Jelle Wesseling ${ }^{1,3}$

${ }^{1}$ Department of Pathology, University Medical Center Groningen, University of Groningen, Groningen, The Netherlands; ${ }^{2}$ Department of Epidemiology, University Medical Center Groningen, University of Groningen, Groningen, The Netherlands and ${ }^{3}$ Department of Pathology, Netherlands Cancer Institute/Antoni van Leeuwenhoek Hospital, Amsterdam, The Netherlands

\begin{abstract}
HER2 overexpression in breast cancer is associated with worse clinical outcome. To select patients for antiHer2-based therapy immunohistochemistry is commonly performed as a first step to assess Her2 status. However, interobserver and interlaboratory variability can significantly compromise adequate assessment of Her2 status. In addition, immunohistochemistry does not always result in an unambiguous test result requiring additional testing for Her2 gene amplification. This study aimed to improve the reliability of Her2 immunohistochemistry by using rabbit monoclonal antibody 4B5 as an alternative to mouse monoclonal antibody CB11 routinely used in our laboratory. Therefore, 283 breast adenocarcinomas were included in a tissue microarray. Immunohistochemistry using the 4B5 and CB11 antibodies, and fluorescence and chromogenic in situ hybridization (FISH or $\mathrm{CISH}$ ) were performed. Immunohistochemistry was scored by two independent investigators. We found that 4B5 staining was more distinct than CB11 staining. For CB11 staining, there were $12 \%(\mathrm{BV})$ and $5 \%(\mathrm{JW}) 2+$ scores compared with $4 \%(\mathrm{BV})$ and $2 \%$ (JW) for $4 \mathrm{~B} 5$. There was a strong trend towards higher interobserver agreement for 4B5 compared with CB11 (4B5: $\kappa$ 0.87, 95\% Cl 0.790.96; CB11: $\kappa 0.77,95 \% \mathrm{Cl} 0.66-0.88)$. There were no significant differences in sensitivity, specificity and predictive values between $C B 11$ and 4B5. Our results indicate that the 4B5 antibody provides more robust assessment of immunohistochemical Her2/neu status and will reduce the number of gene amplification tests compared with $\mathrm{CB} 11$. However, for tumours with a 2 + score additional gene amplification measurement using FISH or CISH remains necessary.

Modern Pathology (2009) 22, 879-886; doi:10.1038/modpathol.2009.37; published online 20 March 2009
\end{abstract}

Keywords: breast carcinoma; immunohistochemistry; validation study; Her2/neu; rabbit monoclonal antibody

The status of the Her2 receptor is an important factor in the prognosis and treatment choice in primary breast carcinoma. ${ }^{1}$ Mortality and recurrence are reduced in patients with Her2/neu-positive breast carcinoma that receive adjuvant therapy with both the humanized anti-Her2/neu monoclonal antibody therapy trastuzumab and chemotherapy. ${ }^{2-5}$

Trastuzumab therapy, however is associated with cardiotoxicity, in $2-4.7 \%$ of patients when used as monotherapy, but in up to $27 \%$ when given concomitantly with anthracycline and cyclophos-

Correspondence: Dr J Wesseling, MD, PhD, Netherlands Cancer Institute/Antoni van Leeuwenhoek Hospital, Plesmanlaan 121, Amsterdam 1066 CX, The Netherlands.

E-mail: j.wesseling@nki.nl

Received 23 January 2009; revised and accepted 17 February 2009; published online 20 March 2009 famide therapy. ${ }^{6}$ Therefore, this treatment is only given to patients with confirmed Her2/neu-positive breast carcinoma and adequate left ventricle ejection fraction. Several methods are used to assess Her2/ neu status in breast cancer. Measurement of gene amplification using fluorescence in situ hybridization (FISH) or chromogenic in situ hybridization (CISH) is considered to be the 'gold standard' in the assessment of Her2/neu status. ${ }^{7-9}$ However, the most commonly used first-line method to determine Her2/neu status is immunohistochemistry. Immunohistochemistry is relatively inexpensive and a routinely used technique in pathology laboratories, which makes it easy to implement. Immunohistochemistry results in a Her2/neu score ranging from 0 (no expression) to $3+$ (strong complete tumour cell membrane expression). ${ }^{10}$ This semiquantitative scoring system does not always result in a clear 
positive or negative Her2/neu amplification status. It is generally agreed that when a Her2 score is ambiguous $(2+$ score $)$ a gene amplification measurement has to be performed. ${ }^{7,11,12}$ Another setback of Her2 immunohistochemistry is the significant interobserver variation and poor interlaboratory reproducibility. ${ }^{13-16}$ As accurate diagnostic assessment of HER2/neu is essential for the appropriate application of trastuzumab-containing treatment regiments, the concordance between immunohistochemistry and the 'gold standard' gene amplification assessment needs to be as high as possible. Therefore, immunohistochemistry staining should be improved to optimize accurate estimation of the HER2/neu status.

In recent years, rabbit monoclonal antibodies have been developed, which show higher affinity and specificity than mouse monoclonal or rabbit polyclonal antibodies resulting in more reliable staining results. ${ }^{17,18}$ To improve Her2 immunohistochemistry reliability in our laboratory, we tested the potential of the rabbit monoclonal antibody 4B5 directed against Her2/neu as an alternative to the mouse monoclonal CB11 directed against Her2/neu, using both a CISH array for Her2/neu and a Her2/neu FISH array as reference.

\section{Materials and methods}

\section{Patients}

To determine the size of the series, a power analysis was performed. In this power analysis, we considered HER2/neu immunohistochemistry negative if the staining pattern and intensity is equivalent to score ' 0 ' or ' $1+$ ' and positive if it is equivalent to score ' $3+$ '. $2+$ cases are not taken into account in this power analysis, because they are clinically uninformative. We assumed 10\% $2+$ cases. We also assumed that the rabbit monoclonal antibody has a better sensitivity and specificity than the mouse monoclonal antibody CB11. Furthermore, a loss of $20 \%$ cases because of unavailability of tumour material or uninterpretability of one or more of the stainings was assumed. On the basis of these assumptions, a sample size of 280 was required to achieve $90 \%$ power to detect an odds ratio of 3.000 using a two-sided McNemar test with a significance level of 0.05000 . The odds ratio is equivalent to a difference between two paired proportions of 0.100 , which occurs when the proportion $\mathrm{T}_{\mathrm{N}}$ negative vs $\mathrm{T}_{\mathrm{O}}$ positive is 0.150 and the proportion $\mathrm{T}_{\mathrm{N}}$ positive vs $\mathrm{T}_{\mathrm{O}}$ negative is 0.050 . A total of 283 consecutive female patients treated for a primary operable invasive carcinoma of the breast at the University Medical Center Groningen between January 2002 and December 2005 were included in this study. Afterwards eight patients were excluded because no representative material was available in the tissue microarray blocks. The analyses have been performed on the resulting group of 275 patients.
Table 1 Patient and tumour characteristics

\begin{tabular}{lr}
\hline & $n(\%)$ \\
\hline $\begin{array}{l}\text { Age at diagnosis } \\
\text { Median (range) }\end{array}$ & $60(29-90)$ \\
Menopausal status & \\
$\quad$ Premenopausal & $78(28)$ \\
Postmenopausal & $197(72)$ \\
Pathological tumour size (mm) & \\
Median (range) & $22(1-140)$ \\
& \\
Pathological tumour type & $109(40)$ \\
IDC & $110(40)$ \\
IDC and DCIS & $25(9)$ \\
ILC & $6(2)$ \\
ILC and LCIS & $25(9)$ \\
other & \\
Grade of differentiation (invasive) & \\
Well & $82(30)$ \\
Moderate & $114(41)$ \\
Poor & $79(29)$ \\
\hline
\end{tabular}

$n$, number of cases; IDC, invasive ductal carcinoma; DCIS, ductal carcinoma in situ; ILC, invasive lobular carcinoma; LCIS, lobular carcinoma in situ; other, other specific subtypes of invasive breast carcinoma.

Patient and tumour characteristics were obtained retrospectively from hospital records and are summarized in Table 1.

\section{Tissue Microarray Construction}

From the patient's tumour paraffin block, three $0.6 \mathrm{~mm}$ core samples of the most representative tumour area were included in a tissue microarray. The technique of tissue microarray production has been described and validated for breast carcinoma by others. ${ }^{19,20}$ In the latter study, authors showed that the concordance between the tissue microarray with the whole tissue sections was over $97 \%$ if three $0.6 \mathrm{~mm}$ core samples per tumour were included in the TMA. In brief, tissue microarrays were compiled as follows: the most representative tumour area was marked on the original haematoxylin and eosin (H\&E)-stained section. Using this section as an orientation, three $0.6 \mathrm{~mm}$ core punches were taken from the selected area in the donor blocks and mounted in a recipient block, using a manual tissue microarray device (Beecher Instruments, Silver Springs, MD, USA). In total, seven tissue microarray blocks, each containing tumour cores from 40 to 50 patients, were made. Using a standard microtome, $3 \mu \mathrm{m}$ sections were cut from these tissue microarray blocks.

\section{Immunohistochemistry, FISH and CISH}

Immunohistochemistry for rabbit monoclonal antibody 4B5 (PATHWAY ${ }^{\circledR}$ HER-2/neu (4B5) rabbit 
monoclonal antibody, Ventana Medical Systems, Illkirch, Cedex, France) and mouse monoclonal antibody CB11 (PATHWAY HER-2/neu (CB11) mouse monoclonal antibody, Ventana Medical Systems) was performed on the tissue microarray sections using the automated Benchmark ${ }^{\circledR}$ platform (Ventana Medical Systems) and according to the manufacturers recommendations. FISH (PathVysion HER-2 DNA Probe Kit, Vysis Inc., Downers Grove, IL, USA) and CISH (SPoT-Light ${ }^{\circledR}$ HER2 CISH $^{\text {TM }}$ Kit, Zymed, Carlsbad, CA, USA) assays were performed according the manufacturers recommendations.

\section{Evaluation of Immunohistochemistry, FISH and CISH}

Scoring of the CB11 and 4B5 immunohistochemistry stainings was performed independently by an experienced pathologist (JW) and a senior resident (BV). Her-2/neu expression was graded as recommended by the HercepTest ${ }^{\mathrm{TM}}$ scoring guidelines: 0 : no staining at all or membrane staining in $<10 \%$ of the tumour cells; $1+$ : a faint/barely perceptible partial membrane staining in $>10 \%$ of the tumour cells; $2+$ : weak-to-moderate complete membrane staining in $>10 \%$ of the tumour cells; $3+$ : strong complete membrane staining in $>10 \%$.

Fluorescence in situ hybridization was scored according to the ASCO guidelines: the ratio of 20 cells was calculated. A ratio $<1.8$ was considered negative, a ratio $>2.2$ was considered positive. For the equivocal cases another 20 cells were counted. In these cases, a ratio 2 was considered positive. A ratio $<2$ was considered negative. For CISH, tumours with at least five signals in more than $50 \%$ of the tumour cells were considered positive.

\section{Data Analysis}

All statistical analyses were performed using the SPSS 14.0 software package.

The feasibility of both staining methods was compared. The number of informative test results and the number of $2+$ scores were calculated for each of the staining methods. Agreement between immunohistochemistry results and in situ hybridization results were calculated in a cross tabulation using a Pearson $\chi^{2}$-test. Sensitivity and specificity were calculated using two methods, including and excluding $2+$ scores. In the first method, $2+$ scores were considered a positive test result. This method is most commonly used in literature and was performed to compare our results to those found in other studies. $2+$ scores are, however, clinically uninformative and were therefore eliminated from analysis in the second method. The positive predictive value (PPV) was calculated by dividing the number of cases in the immunohistochemistry $3+$ group with amplification on FISH or CISH by the total number of cases with an immunohistochemistry $3+$ score. The negative predictive value (NPV) was calculated by dividing the number of cases immunohistochemistry score 0 or $1+$ without amplification on FISH or CISH by the total number of patients with an immunohistochemistry 0 or $1+$ score. For sensitivity, specificity and predictive values a $95 \% \mathrm{CI}$ interval was calculated. Interobserver agreement was calculated in a cross tabulation using Cohen's $\kappa$-test.

\section{Results}

\section{Feasibility}

In Figure 1, an example of both a 4B5 and a CB11 staining for 1 case is shown. In general, the 4B5 staining was more distinct compared with the CB11 staining. Also the 4B5 staining showed less nonspecific cytoplasmic staining.

\section{Test Characteristics}

Of the 275 cases included, 262 (95\%) of the cases were adequately represented in the tissue micro-
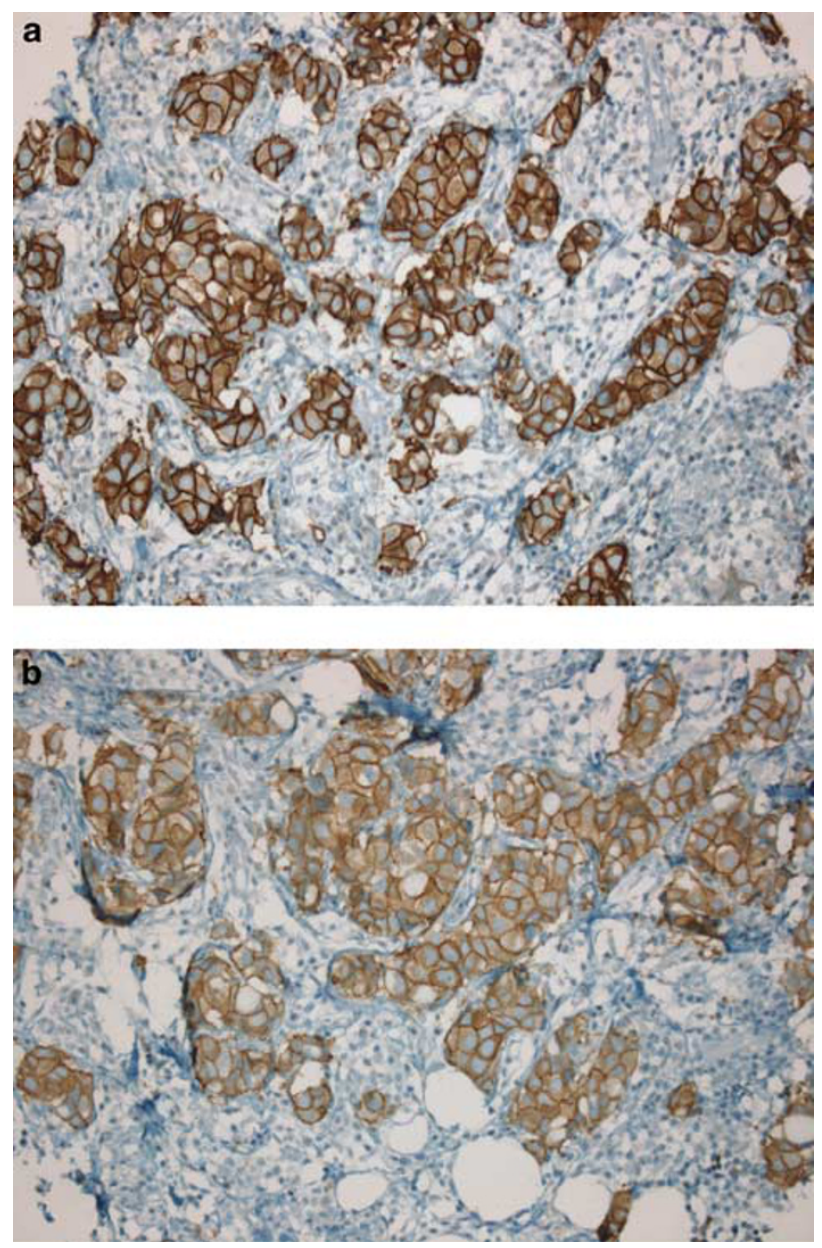

Figure 1 Comparison of $3+$ case for 4B5 and CB11. (a) 4 B5. (b) CB11. 
Table 2 Correlation of 4B5 and CB11 for observer BV (a) and JW (b)

\begin{tabular}{|c|c|c|c|c|c|}
\hline \multicolumn{6}{|c|}{$C B 11 B V$} \\
\hline $4 B 5 B V$ & 0 & 1 & 2 & 3 & Total \\
\hline 0 & 149 (95) & $20(50)$ & 4 (14) & $0(0)$ & $173(71)$ \\
\hline 1 & $7(4)$ & $19(48)$ & $14(50)$ & $0(0)$ & 40 (16) \\
\hline 2 & 1 (1) & $1(2)$ & 8 (29) & $0(0)$ & $13(4)$ \\
\hline 3 & $0(0)$ & $0(0)$ & $2(7)$ & 19 (100) & 21(9) \\
\hline $\begin{array}{l}\text { Total } \\
\chi^{2}: P \leq 0.001\end{array}$ & 157 (100) & 40 (100) & $28(100)$ & 19 (100) & 244 (100) \\
\hline \multicolumn{6}{|l|}{ (b) } \\
\hline & \multicolumn{5}{|c|}{ CB11 JW } \\
\hline $4 B 5 J W$ & 0 & 1 & 2 & 3 & Total \\
\hline 0 & 106 (96) & $28(35)$ & $0(0)$ & $0(0)$ & 134 (60) \\
\hline 1 & $5(4)$ & $51(64)$ & $6(50)$ & $0(0)$ & $62(28)$ \\
\hline 2 & $0(0)$ & $1(1)$ & $4(33)$ & $0(0)$ & $5(2)$ \\
\hline 3 & $0(0)$ & $0(0)$ & 2 (17) & $20(100)$ & $22(10)$ \\
\hline Total & $111(100)$ & 80 (100) & $12(100)$ & $20(100)$ & $223(100)$ \\
\hline
\end{tabular}

$\chi^{2}: P \leq 0.001$

CB11: mouse monoclonal antibody CB11; 4B5: rabbit monoclonal antibody 4B5; BvdV: Bert van der Vegt; JW: Jelle Wesseling.

array. Immunohistochemistry could be evaluated in 83 (JW) and 90\% (BV) of the cases for 4B5, in 87 (JW) and $91 \%(B V)$ of the cases for CB11. Differences in evaluation percentages between authors have been caused by disagreement on the overall percentage of tumour available in the core punches and the relative percentages of invasive tumour vs in situ tumour available in the core punches. FISH could be evaluated in $90 \%$ and $\mathrm{CISH}$ in $92 \%$ of the cases.

\section{Agreement}

In 230 of the 240 assessable cases (96\%), there was agreement between FISH and CISH scoring ( $\kappa 0.837$, 95\% CI 0.737-0.937). There was an agreement of $80(\mathrm{BV})$ and $81 \%$ (JW) between the scoring results of 4B5 and CB11 (Table 2). The disagreement between scores is mainly caused by the $2+$ scores. There was a reduction of more than $50 \%$ for the number of cases scored as $2+$ when comparing CB11 with 4B5, respectively, 28 cases $(12 \%)$ vs 13 cases (4\%) (BV) and 12 cases $(5 \%)$ vs 5 cases ( $2 \%)$ (JW). When excluding the $2+$ cases from analysis, there was no difference between both antibodies for the classification of cases as amplified or not amplified (McNemar's test $P=1.0$, data not shown) eg, there would be no clinical consequences when using either of the antibodies. In Tables 3 and 4, the results for the concordance between immunohistochemistry and respectively FISH and CISH are shown. Sensitivity (including and excluding $2+$ scores), specificity (including and excluding $2+$ scores), PPV and NPV of 4B5 and CB11 using
Table 3 comparison of immunohistochemistry with FISH

\begin{tabular}{|c|c|c|c|}
\hline & Amplification & No amplification & Total \\
\hline \multicolumn{4}{|c|}{$I H C$ CB11 BvdV } \\
\hline 0 & $3(8)$ & $150(74)$ & $153(64)$ \\
\hline 1 & $2(5)$ & 38 (19) & 40 (16) \\
\hline 2 & $13(35)$ & $15(7)$ & $28(12)$ \\
\hline 3 & $19(52)$ & $0(0)$ & $19(8)$ \\
\hline Total & $37(100)$ & $203(100)$ & $240(100)$ \\
\hline \multicolumn{4}{|c|}{$\chi^{2}: P \leq 0.001$} \\
\hline \multicolumn{4}{|c|}{$I H C 4 B 5 B v d V$} \\
\hline 0 & $5(14)$ & $165(81)$ & $170(71)$ \\
\hline 1 & $4(11)$ & 36 (18) & $40(17)$ \\
\hline 2 & 7 (19) & $3(1)$ & $10(4)$ \\
\hline 3 & $21(56)$ & $0(0)$ & $21(8)$ \\
\hline Total & $37(100)$ & $204(100)$ & $241(100)$ \\
\hline$\chi^{2}: P \leq$ & & & \\
\hline \multicolumn{4}{|c|}{ IHC CB11 JW } \\
\hline 0 & $2(5)$ & 116 (59) & $118(51)$ \\
\hline 1 & $5(14)$ & $78(40)$ & $83(36)$ \\
\hline 2 & $10(27)$ & $2(1)$ & $12(5)$ \\
\hline 3 & $20(54)$ & $0(0)$ & $20(8)$ \\
\hline Total & 37 (100) & $196(100)$ & $233(100)$ \\
\hline \multicolumn{4}{|c|}{$\chi^{2}: P \leq 0.001$} \\
\hline \multicolumn{4}{|c|}{ IHC $4 B 5 \mathrm{JW}$} \\
\hline 0 & $3(8)$ & 131 (69) & $134(60)$ \\
\hline 1 & $7(20)$ & $57(30)$ & $64(28)$ \\
\hline 2 & $4(11)$ & $1(1)$ & $5(2)$ \\
\hline 3 & $22(61)$ & $0(0)$ & $22(10)$ \\
\hline Total & $36(100)$ & 189 (100) & $225(100)$ \\
\hline$\chi^{2}: P \leq$ & & & \\
\hline
\end{tabular}

IHC: immunohistochemistry; CB11: mouse monoclonal antibody CB11; 4B5: rabbit monoclonal antibody 4b5; BvdV: Bert van der Vegt; JW: Jelle Wesseling. 
both FISH and CISH as reference are shown in Table 5. Sensitivity, specificity, PPV and NPV did not show any significant differences between both

Table 4 comparison of immunohistochemistry with CISH

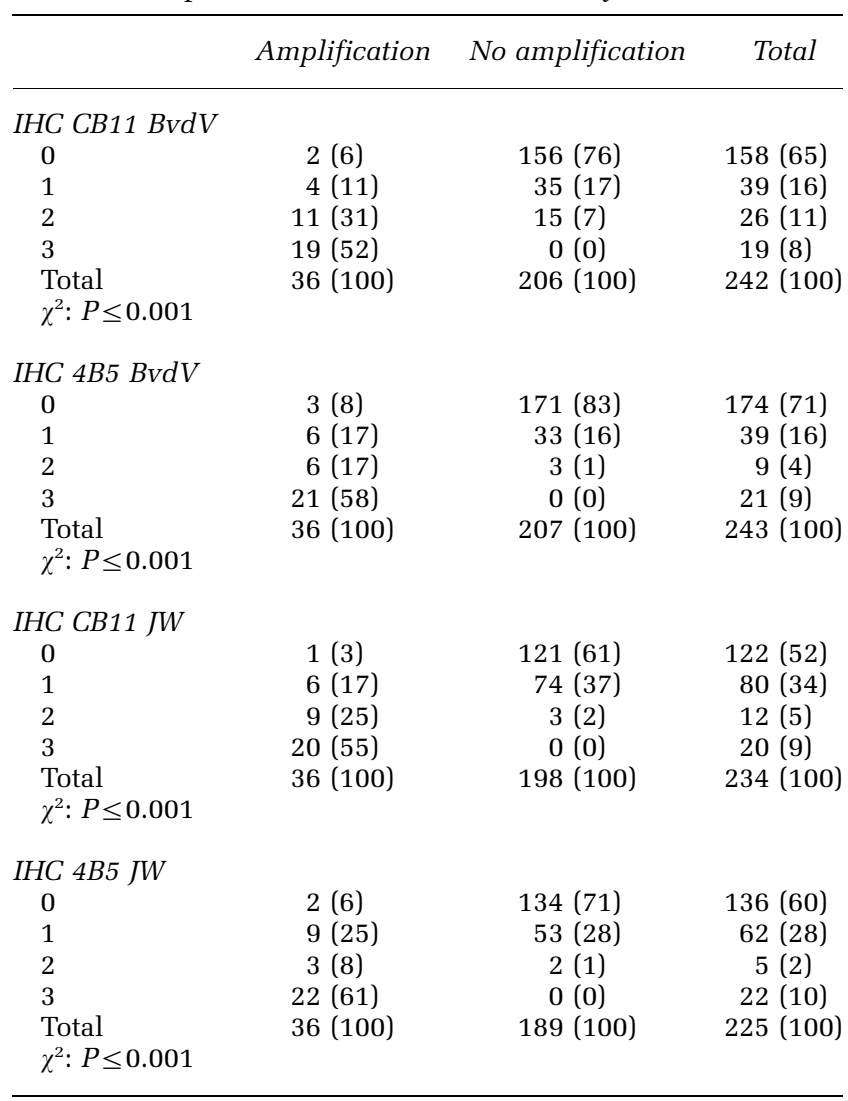

4B5, rabbit monoclonal antibody 4B5; BvdV, Bert van der Vegt; CB11, mouse monoclonal antibody CB11; IHC, immunohistochemistry; JW, Jelle Wesseling. antibodies and between both observers. However, there was a near significant difference in specificity (including $2+$ scores) for the lesser experienced observer (BV) between 4B5 and CB11: (4B5: 0.99, 95\% CI 0.95-1.0; CB11: 0.93, 95\% CI 0.88-0.96). Although not significant, there was a trend towards an increased concordance between observers for 4B5 compared with CB11 (4B5: $\kappa$ 0.87, 95\% CI 0.79-0.96; CB11: $\kappa$ 0.77, 95\% CI 0.66-0.88) (Table 6).

\section{Discussion}

In this study, we tested the potential of the 4B5 antiHer2 rabbit monoclonal antibody by comparing it with the CB11 anti-Her2 mouse monoclonal antibody on a consecutive series of invasive breast adenocarcinomas, using both FISH and CISH as reference. We found that 4B5 staining was more distinct and showed less nonspecific cytoplasmic background staining, which led to a more than $50 \%$ reduction of the number of $2+$ scores for $4 \mathrm{~B} 5$ compared with CB11. In this well powered study we found no significant differences in sensitivity, specificity, predictive values and interobserver concordance between 4B5 and CB11. However, there was a trend towards a higher specificity for 4B5 for one of the observers (BV) and a trend towards an increased interobserver concordance for 4B5. The - nonsignificant - differences in the test results of

Table 6 Concordance between observers

\begin{tabular}{llc}
\hline & $\kappa$ & $95 \% C I$ \\
\hline 4B5 & 0.87 & $0.79-0.96$ \\
CB11 & 0.77 & $0.66-0.88$ \\
\hline
\end{tabular}

Table 5 sensitivity, specificity and predictive values for immunohistochemistry compared with FISH and CISH

\begin{tabular}{|c|c|c|c|c|c|c|c|}
\hline & \multicolumn{7}{|c|}{ FISH } \\
\hline & Sensitivity & Specificity & $\begin{array}{c}\text { Sensitivity } \\
\text { (excl 2+ scores) }\end{array}$ & $\begin{array}{c}\text { Specificity } \\
\text { (excl 2+ scores) }\end{array}$ & $P P V$ & $N P V$ & $\% 2+$ \\
\hline 4B5 BvdV & $0.75(0.57-0.87)$ & $0.99(0.95-1.0)$ & $0.70(0.50-0.85)$ & $1.0(0.98-1.0)$ & $1.0(0.81-1.0)$ & $0.96(0.92-0.98)$ & 4 \\
\hline CB11 BvdV & $0.83(0.67-0.93)$ & $0.93(0.88-0.96)$ & $0.79(0.57-0.92)$ & $1.0(0.98-1.0)$ & $1.0(0.79-1.0)$ & $0.97(0.94-0.99)$ & 12 \\
\hline 4B5 JW & $0.69(0.52-0.83)$ & $0.99(0.96-1.0)$ & $0.69(0.50-0.83)$ & $1.0(0.98-1.0)$ & $1.0(0.82-1.0)$ & $0.95(0.91-0.97)$ & 2 \\
\hline \multirow[t]{3}{*}{ CB11 JW } & $0.81(0.63-0.91)$ & $0.99(0.96-1.0)$ & $0.74(0.53-0.88)$ & $1.0(0.98-1.0)$ & $1.0(0.80-1.0)$ & $0.97(0.93-0.98)$ & 5 \\
\hline & \multicolumn{7}{|c|}{$\mathrm{CISH}$} \\
\hline & Sensitivity & Specificity & $\begin{array}{c}\text { Sensitivity } \\
\text { (excl 2+ scores) }\end{array}$ & $\begin{array}{c}\text { Specificity } \\
\text { (excl 2+ scores) }\end{array}$ & $P P V$ & $N P V$ & $\% 2+$ \\
\hline 4B5 BvdV & $0.76(0.58-0.88)$ & $0.99(0.95-1.0)$ & $0.70(0.50-0.85)$ & $1.0(0.98-1.0)$ & $1.0(0.81-1.0)$ & $0.96(0.92-0.98)$ & 4 \\
\hline CB11 BvdV & $0.86(0.70-0.95)$ & $0.93(0.88-0.96)$ & $0.76(0.54-0.90)$ & $1.0(0.98-1.0)$ & $1.0(0.80-1.0)$ & $0.97(0.93-0.99)$ & 11 \\
\hline $4 \mathrm{~B} 5 \mathrm{JW}$ & $0.72(0.55-0.85)$ & $0.99(0.96-1.0)$ & $0.67(0.48-0.81)$ & $1.0(0.97-1.0)$ & $1.0(0.82-1.0)$ & $0.94(0.90-0.97)$ & 2 \\
\hline CB11 JW & $0.81(0.64-0.91)$ & $0.99(0.96-1.0)$ & $0.74(0.53-0.88)$ & $1.0(0.98-1.0)$ & $1.0(0.80-1.0)$ & $0.97(0.93-0.98)$ & 5 \\
\hline
\end{tabular}

4B5, rabbit monoclonal antibody 4b5; BvdV, Bert van der Vegt; CB11, mouse monoclonal antibody CB11; IHC, immunohistochemistry; JW, Jelle Wesseling; NPV, negative predictive value; PPV, positive predictive value. 
both observers would have no consequences for clinical decision making regarding the Her2 status of the carcinomas investigated.

Gene amplification measurement is considered to be the 'gold standard' for Her2/neu status assessment, ${ }^{7-9}$ although it suffers from variability between institutes ${ }^{21,22,}$ and the sensitivity varies between detection methods used. ${ }^{9}$ Traditionally, gene amplification measurement has been performed using FISH assays for Her2/neu gene amplification. This method, however, has several disadvantages: FISH is expensive, is time demanding, ${ }^{8}$ is not readily accommodated in most pathology laboratories and is accompanied by technical challenges. ${ }^{23}$ More recently CISH assays have been developed. Instead of the fluorogens used in FISH, this technique uses chromogens. This has several advantages ${ }^{24}$ : where FISH requires a fluorescence microscope for interpretation, CISH can be interpreted using a normal bright field microscope. CISH allows analysis of tumour morphology, making it possible to interpret tumour heterogeneity and gene copy number in different components of the tumour (an invasive and an in situ component). Also FISH signals are labile and fade over time, but CISH produces a permanent staining.

Many authors have compared CISH with FISH (reviewed by Lambros et $a^{24}$ ). Most studies find an agreement of both methods of more than $90 \%$. In a multicenter study, where pathology laboratories blindly performed CISH on cases from each other this method was validated. ${ }^{25}$ That study also reported an intra- and interobserver agreement of over $90 \%$ and concluded that CISH is a viable alternative to FISH. In this study, the agreement between FISH and CISH was $96 \%$, which is in line with the conclusions of those earlier studies. Of the 10 discordant cases in our series 5 cases contained an in situ component. This tumour heterogeneity might have led to the discordance because distinction between the invasive and the in situ component can be difficult in FISH where tumour morphology is not readily recognized in all cases.
Most pathology laboratories use immunohistochemistry to assess Her2/neu status. Immunohistochemistry is not only less expensive than in situ methods, there is also much experience with immunohistochemistry, which makes it a method that is easily implemented in the daily practice of pathology laboratories. Immunohistochemistry, however, has several disadvantages: immunohistochemistry is scored semiquantitively, which leads to a $0-3+$ score rather than to a clear amplification/ no amplification outcome. Scores 0 and $1+$ are considered non-amplified and score $3+$ is considered amplified. An ideal Her2 antibody has a low number of indeterminate $(2+)$ cases and a high PPV and NPV. We found that 4B5 staining compared with CB11 was more distinct and showed less nonspecific cytoplasmic background staining. This has led to an increase in interobserver concordance (4B5: $\kappa 0.87,95 \%$ CI 0.79-0.96; CB11: $\kappa 0.77,95 \%$ CI $0.66-0.88$ ) that showed a trend towards significance. Interobserver concordance is essential because it has far reaching consequences for the choice of therapy regimen and response to therapy. A few studies have assessed interobserver concordance for CB11. Two of those studies found an interobserver concordance of 0.74 , which is comparable with our results $(0.77){ }^{13,15}$ In a study by Tsuda $e a^{16}$ an interobserver concordance of only 0.29 was found for CB11. However, this study used a different method to calculate concordance. The concordance of 0.87 that we found for $4 \mathrm{~B} 5$ is considered almost perfect, ${ }^{26}$ which underlines that 4B5 is a safe method to assess Her2 status in breast cancer. To our knowledge, no other studies have assessed interobserver concordance for 4B5. Interlaboratory concordance was not assessed in this study. One study mentions a perfect interlaboratory concordance on a very limited number of cases. ${ }^{27}$ Future studies are needed to assess this issue.

In this series, a cutoff of $10 \%$ staining was used for scoring Her2 immunohistochemistry. This cutoff was chosen based on Dutch guidelines, which differ from the ASCO guideline using a cutoff of $30 \%$.

Table 7 Sensitivity, specificity, predictive and concordance for CB11 from literature

\begin{tabular}{|c|c|c|c|c|c|c|}
\hline Author & Study size & Sensitivity & Specificity & Method & $P P V$ & $N P V$ \\
\hline Kakar et al ${ }^{30}$ & 112 & $0.93(0.66-1.0)^{\mathrm{a}}$ & $0.98(0.90-1.0)^{\mathrm{a}}$ & 2 & $0.88(0.60-0.98)^{\mathrm{a}}$ & $0.99(0.92-1.0)^{\mathrm{a}}$ \\
\hline Bartlett et $a l^{13}$ & 213 & $0.85(0.70-0.94)^{\mathrm{a}}$ & $0.69(0.62-0.76)^{\mathrm{a}}$ & 1 & $0.40(0.30-0.51)^{\mathrm{a}}$ & $0.95(0.89-0.98)^{\mathrm{a}}$ \\
\hline Press et $a l^{9}$ & 74 & $0.721(0.56-0.85)$ & $1.00(0.95-1.0)$ & 1 & & \\
\hline Press et $a l^{21}$ & 64 & $0.95(0.74-1.0)$ & $0.84(0.70-0.93)$ & 1 & & \\
\hline Ricardo et $a l^{31}$ & 190 & $0.52(0.37-0.67)^{\mathrm{a}}$ & $0.98(0.93-1.0)^{\mathrm{a}}$ & 2 & $0.92(0.72-0.99)^{\mathrm{a}}$ & $0.83(0.75-0.89)^{\mathrm{a}}$ \\
\hline \multirow[t]{2}{*}{ Powell et $a l^{27}$} & $178^{\mathrm{b}}$ & $0.92(0.83-0.96)^{\mathrm{a}}$ & $0.91(0.82-0.96)^{\mathrm{a}}$ & 1 & $0.92(0.83-0.96)^{\mathrm{a}}$ & $0.91(0.82-0.96)^{\mathrm{a}}$ \\
\hline & $144^{\mathrm{b}}$ & $0.74(0.64-0.83)^{\mathrm{a}}$ & $0.91(0.80-0.97)^{\mathrm{a}}$ & 1 & $0.93(0.83-0.97)^{\mathrm{a}}$ & $0.71(0.59-0.80)^{\mathrm{a}}$ \\
\hline Egervari et $a 2^{29}$ & 199 & $0.83(0.58-0.96)^{\mathrm{a}}$ & $0.99(0.96-1.0)^{\mathrm{a}}$ & 2 & $0.94(0.68-1.0)^{\mathrm{a}}$ & $0.98(0.94-0.99)^{\mathrm{a}}$ \\
\hline
\end{tabular}

Method 1,2+ and 3+ scores considered positive; Method 2, 2+ cases excluded from analysis; PPV, positive predictive value; NPV, negative predictive value.

${ }^{\mathrm{a}}$ Calculated from the data available in the article.

${ }^{\mathrm{b}}$ Two separate subsets were studied in the article. 
However, this difference in cutoff did not cause a significant change in the results.

The number of cases that were scored as $2+$ was lower for 4B5. For 4B5, observers scored 4 and $2 \% 2$ + cases; for CB11 this was 12 and 5\%. The number of CB11 $2+$ cases in this study is comparable the with numbers in literature, where the number of cases scored $2+$ using an mouse monoclonal antibody ranges from 2 to $20.5 \%$, and is usually around $10 \% .{ }^{28}$ We expect that the introduction of $4 \mathrm{~B} 5$ will reduce the number of FISH or CISH assays that will have to be performed, which leads to a reduction in costs. In this well powered study, this decrease of indeterminate cases did not lead to a significant difference in sensitivity, specificity, or predictive power between CB11 and 4B5. Small numbers did not allow a statistically reliable subgroup analysis to assess differences in sensitivity, specificity and predictive values for the immunohistochemistry $2+$ cases of both antibodies using FISH or CISH as a reference. In table 7 , the results from other studies assessing sensitivity, specificity and predictive values of CB11 are shown. Most studies considered $2+$ cases to be amplified for the calculations. As discussed before $2+$ cases correlate with gene amplification very poorly. ${ }^{7,11,12}$ We believe that $2+$ cases should be excluded from analysis when assessing sensitivity, specificity and predictive values. The values for sensitivity, specificity and predictive values that we found in this study are comparable with those found in literature. Powell et $a l^{27}$ performed a study comparing 4B5 with CB11 using FISH as a reference in two subsets of cases, one containing samples from a single institution, and one containing samples from a multicenter tissue bank. In that study the levels of sensitivity were higher and the levels of specificity were lower than in this study. However, these differences were not significant for the subset best comparable with this study group, ie, the single institution subset. PPV and NPV show no significant difference between both antibodies, which underlines that the lower number of indeterminate cases which led to the increase in sensitivity and specificity did not cause a loss of predictive value of the test. In a group of 199 invasive breast cancers, Egervari et al ${ }^{29}$ compared the $4 \mathrm{~B} 5$ antibody with a number of antibodies including the CB11 antibody using FISH as a reference. When recalculating their results using the method described earlier they had sensitivity (0.76 (0.5-0.92)), specificity (0.99 (0.96-1.0)), PPV (0.93 (0.64-1.0)) and NPV (0.97 (0.93-0.99)) that does not differ significantly to our results. Although they suggest a lower sensitivity of 4B5 compared with CB11, this result is not significant (Table 7).

In conclusion, we showed that the novel 4B5 rabbit monoclonal anti-Her2/neu antibody has a good agreement with both FISH and CISH and has a sensitivity, a specificity and predictive values comparable with CB11. We found a reduction of more than $50 \%$ in the number of indeterminate cases for
4B5 and an increase in interobserver concordance. Our results indicate that immunohistochemistry using the 4B5 antibody provides more robust and hence more reliable assessment of the Her2/neu status detection and will reduce the number of gene amplification tests compared with the CB11 antibody. However, for patients with a $2+$ score additional gene amplification measurement using FISH or CISH remains mandatory.

\section{Acknowledgement}

The 4B5 and CB11 reagents were kindly provided by Ventana Medical Systems, Illkirch, France.

\section{Disclosure/conflict of interest}

None.

\section{References}

1 Goldhirsch A, Glick JH, Gelber RD, et al. Meeting highlights: international expert consensus on the primary therapy of early breast cancer 2005. Ann Oncol 2005));16:1569-1583.

2 Romond EH, Perez EA, Bryant J, et al. Trastuzumab plus adjuvant chemotherapy for operable her2-positive breast cancer. N Engl J Med 2005;353:1673-1684.

3 Joensuu $\mathrm{H}$, Kellokumpu-Lehtinen $\mathrm{P}$, Bono $\mathrm{P}$, et al. Adjuvant docetaxel or vinorelbine with or without trastuzumab for breast cancer. N Engl J Med 2006;354: 809-820.

4 Robert N, Leyland-Jones B, Asmar L, et al. Randomized phase III study of trastuzumab, paclitaxel, and carboplatin compared with trastuzumab and paclitaxel in women with HER-2-overexpressing metastatic breast cancer. J Clin Oncol 2006;24:2786-2792.

5 Smith I, Procter M, Gelber RD, et al. 2-year follow-up of trastuzumab after adjuvant chemotherapy in her2positive breast cancer: a randomised controlled trial. Lancet 2007;369:29-36.

6 Bird BRJH, Swain SM. Cardiac toxicity in breast cancer survivors: review of potential cardiac problems. Clin Cancer Res 2008;14:14-24.

7 Dowsett M, Bartlett J, Ellis IO, et al. Correlation between immunohistochemistry (herceptest) and fluorescence in situ hybridization (fish) for her-2 in 426 breast carcinomas from 37 centres. J Pathol 2003; 199:418-423.

8 Yaziji H, Goldstein LC, Barry TS, et al. Her-2 testing in breast cancer using parallel tissue-based methods. JAMA 2004;291:1972-1977.

9 Press MF, Slamon DJ, Flom KJ, et al. Evaluation of her-2/neu gene amplification and overexpression: comparison of frequently used assay methods in a molecularly characterized cohort of breast cancer specimens. J Clin Oncol 2002;20:3095-3105.

10 Wolff AC, Hammond MEH, Schwartz JN, et al. American society of clinical oncology/college of american pathologists guideline recommendations for human epidermal growth factor receptor 2 testing in breast cancer. Arch Pathol Lab Med 2007;131:18. 
11 Lebeau A, Deimling D, Kaltz C, et al. Her-2/neu analysis in archival tissue samples of human breast cancer: comparison of immunohistochemistry and fluorescence in situ hybridization. J Clin Oncol 2001;19:354-363.

12 Fornier M, Risio M, Van Poznak C, et al. Her2 testing and correlation with efficacy of trastuzumab therapy. Oncology (Williston Park) 2002;16:1340-1348, 1351-2; discussion 1352, 1355-8.

13 Bartlett JM, Going JJ, Mallon EA, et al. Evaluating her2 amplification and overexpression in breast cancer. J Pathol 2001;195:422-428.

14 Gancberg D, Järvinen T, di Leo A, et al. Evaluation of her-2/neu protein expression in breast cancer by immunohistochemistry: an interlaboratory study assessing the reproducibility of her-2/neu testing. Breast Cancer Res Treat 2002;74:113-120.

15 Thomson TA, Hayes MM, Spinelli JJ, et al. Her-2/ neu in breast cancer: interobserver variability and performance of immunohistochemistry with 4 antibodies compared with fluorescent in situ hybridization. Mod Pathol 2001;14:1079-1086.

16 Tsuda H, Sasano H, Akiyama F, et al. Evaluation of interobserver agreement in scoring immunohistochemical results of her-2/neu (c-erbb-2) expression detected by herceptest, nichirei polyclonal antibody, cb11 and tab250 in breast carcinoma. Pathol Int 2002;52:126-134.

17 Gouvêa AP, Milanezi F, Olson SJ, et al. Selecting antibodies to detect her2 overexpression by immunohistochemistry in invasive mammary carcinomas. Appl Immunohistochem Mol Morphol 2006;14:103-108.

18 Rossi S, Laurino L, Furlanetto A, et al. Rabbit monoclonal antibodies: a comparative study between a novel category of immunoreagents and the corresponding mouse monoclonal antibodies. Am J Clin Pathol 2005;124:295-302.

19 Kononen J, Bubendorf L, Kallioniemi A, et al. Tissue microarrays for high-throughput molecular profiling of tumor specimens. Nat Med 1998;4:844-847.

20 Camp R, Charette L, Rimm D. Validation of tissue microarray technology in breast carcinoma. Lab Invest 2000;80:1943-1949.

21 Press MF, Sauter G, Bernstein L, et al. Diagnostic evaluation of her-2 as a molecular target: an assessment of accuracy and reproducibility of laboratory testing in large, prospective, randomized clinical trials. Clin Cancer Res 2005;11:6598-6607.

22 Dybdal N, Leiberman G, Anderson S, et al. Determination of her2 gene amplification by fluorescence in situ hybridization and concordance with the clinical trials immunohistochemical assay in women with metastatic breast cancer evaluated for treatment with trastuzumab. Breast Cancer Res Treat 2005;93:3-11.

23 Bartlett JMS. Fluorescence in situ hybridization: technical overview. Methods Mol Med 2004;97:77-87.

24 Lambros MBK, Natrajan R, Reis-Filho JS. Chromogenic and fluorescent in situ hybridization in breast cancer. Hum Pathol 2007;38:1105-1122.

25 van de Vijver M, Bilous M, Hanna W, et al. Chromogenic in situ hybridisation for the assessment of her2 status in breast cancer: an international validation ring study. Breast Cancer Res 2007;9:R68.

26 Landis JR, Koch GG. The measurement of observer agreement for categorical data. Biometrics 1977;33: 159-174.

27 Powell WC, Hicks DG, Prescott N, et al. A new rabbit monoclonal antibody (4b5) for the immunohistochemical (ihc) determination of the her2 status in breast cancer: comparison with cb11, fluorescence in situ hybridization (fish), and interlaboratory reproducibility. Appl Immunohistochem Mol Morphol 2007;15:94-102.

28 Dendukuri N, Khetani K, McIsaac M, et al. Testing for her2-positive breast cancer: a systematic review and cost-effectiveness analysis. CMAJ 2007;176:1429-1434.

29 Egervari K, Szollosi Z, Nemes Z. Immunohistochemical antibodies in breast cancer her2 diagnostics. a comparative immunohistochemical and fluorescence in situ hybridization study. Tumour Biol 2008;29:18-27.

30 Kakar S, Puangsuvan N, Stevens JM, et al. Her-2/neu assessment in breast cancer by immunohistochemistry and fluorescence in situ hybridization: comparison of results and correlation with survival. Mol Diagn 2000;5:199-207.

31 Ricardo SAV, Milanezi F, Carvalho ST, et al. Her2 evaluation using the novel rabbit monoclonal antibody sp3 and cish in tissue microarrays of invasive breast carcinomas. J Clin Pathol 2007;60:1001-1005. 